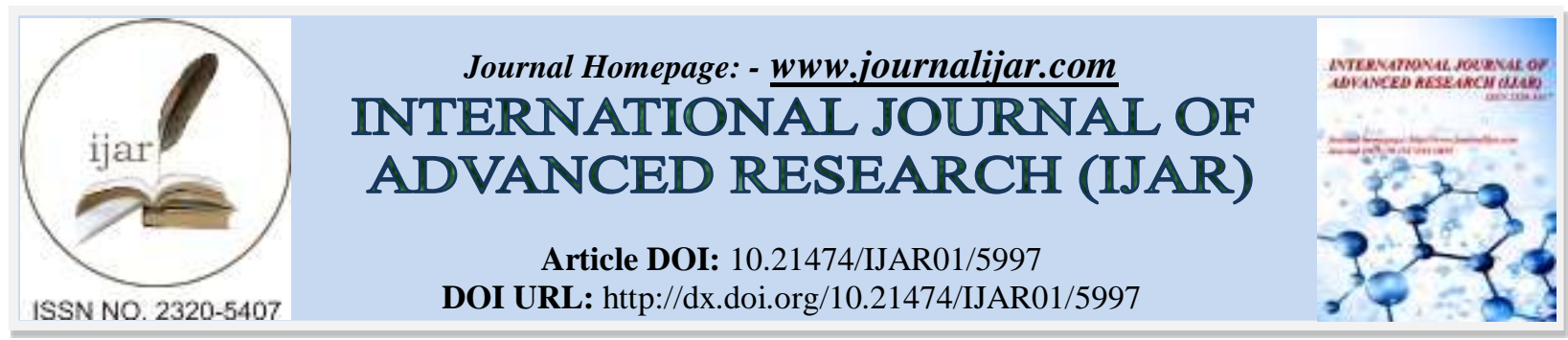

RESEARCH ARTICLE

\title{
A STUDY OF CORRELATION BETWEEN DIFFERENT SITES OF URINE SAMPLING, INCLUDING STONES AS PREDICTORS OF UROSEPSIS FOLLOWING PERCUTANEOUS NEPHROLITHOTOMY.
}

\author{
A. V. Venugopal, Shanmugha Das.K.V, Edikkula Varghese, N. Madhavan, Manikandan, A. T. Rajeevan And \\ Felix Cardoza. \\ From Government Medical College, Kozhikode, Kerala, India.
}

\section{Manuscript Info}

Manuscript History

Received: 07 October 2017

Final Accepted: 09 November 2017

Published: December 2017

Key words:-

Urosepsis, Renal calculus culture, Pelvic urine culture, PCNL sepsis.

\section{Abstract}

Aim: To determine the correlation between urine sampling from different sites, including stones, and also to ascertain which is more predictive of urosepsis.

Material and Methods: All patients undergoing PCNL in Government Medical College Hospital, Calicut during the period of one year (from March 2016 to February 2017) who fulfilled our selection criteria were recruited. The samples collected were 1) midstream urine and bladder urine at cystoscopy, 2) renal pelvic urine collected at the time of ureteric catheter insertion 3) renal pelvic urine collected at percutaneous puncture of the pelvicaliceal system and 4) stones which were extracted. The samples were sent immediately for C\&S. Patients were monitored for systemic inflammatory response sysndrome (SIRS).

Results: Total of 135 cases were suitable for analysis. Midstream urine C\&S was positive in $9.63 \%$ of cases. Stone C\&S was positive in $30.37 \%$ and pelvic C\&S was positive in $20.74 \%$. Pelvic urine C\&S was a better predictor of infected stones than bladder urine C\&S. Of the 135 patients $31.9 \%$ had SIRS and 4 patients experienced septic shock. Patients with infected stones or pelvic urine were found to be at a relative risk for urosepsis that was at least 10 times greater $(\mathrm{p}<0.0001)$. Bladder urine was not a good predictor of SIRS. Stone C\&S had a positive predictive value of 0.7 , which was the highest. Preoperative dilatation of pelvicalyceal system correlated with infected pelvic urine. None of the patients with urosepsis had positive blood C\&S.

Conclusions: Positive stone $C \& S$ and pelvic urine $C \& S$ are better predictors of urosepsis than bladder urine in our study. So, routine collection of pelvic urine and stone for culture and sensitivity is recommended.

\section{Introduction:-}

The most frequently performed intrarenal surgery for stone disease at our institution is percutaneous nephrolithotomy (PCNL). Indications for this procedure are failed extracorporeal shock wave lithotripsy, larger stones (greater than $2.5 \mathrm{~cm}$ ) and stones in anatomically abnormal kidneys. Despite sterile preoperative urine and prophylactic antibiotics, septicemia during or following the procedure can be catastrophic. ${ }^{1,2}$ If bladder urine taken 
one week prior to surgery is sterile, our practice has been to operate, giving a single dose of gentamicin at anesthesia induction. The procedure is deferred until sterile urine is achieved if midstream urine (MSU) is infected. Despite all this, patients still can have life threatening systemic infections. Infected stones, obstructed kidneys, prolonged manipulation, difficult access and comorbidity have been held responsible for this complication. ${ }^{3}$ This often results in high dependence or intensive care that escalates the total cost of treatment.

In this prospective study we analysed various culture specimens, namely bladder urine, renal pelvic urine and fragmented stones, and identified which is most predictive of urosepsis. We also identified other preoperatively determinable factors that might contribute to systemic infection in post operative period.

\section{Materials and Methods:-}

We performed this prospective clinical study in all our patients undergoing PCNL between March 1, 2016 and February 28, 2017. The study exclusion criteria are listed in Appendix.

One day prior to surgery a MSU or a clean, straight catheter specimen of urine was sent for culture and sensitivity testing (C\&S). Patients were given $5 \mathrm{mg} / \mathrm{kg}$ body weight gentamicin intravenously just before anesthesia induction, according to our standard protocol.

After antiseptic preparation with chlorhexidine, a standard cystoscope with a working channel was introduced into the bladder and a urine sample was collected for C\&S. The opposite ureterovesical opening was cannulated with a sterile 0.035 inch straight guide wire and under image intensification it was passed into the pelvicaliceal system. A sterile 6Fr ureteral catheter was then threaded on the guide wire until the tip was confirmed to lie within the collecting system and urine was collected from the system and sent for C\&S as pelvic urine C\&S (PUC 3). This ureteric catheter was then removed. The ipsilateral ureterovesical opening was cannulated with a sterile $0.035-$ inch straight guide wire and under image intensification it was passed into the pelvicaliceal system. A sterile 6Fr ureteral catheter was then threaded on the guide wire until the tip was confirmed to lie within the collecting system and urine was collected from the system and sent for C\&S as pelvic urine C\&S (PUC 2).

The patient was then turned prone and sterile preparation was carried out. Percutaneous access into the ipsilateral pelvicaliceal system was achieved under image (x-ray fluoroscopy) intensification using a fine, 16 gauge Kellet needle. Urine from the pelvicaliceal system was first aspirated and the sample sent as pelvic urine C\&S (PUC 1).

The tract was then dilated using Amplatz or Alken coaxial fascial dilators until a 30Fr Amplatz sheath could be placed into the pelvicaliceal system. Nephroscopy and pneumatic lithotripsy were performed under low pressure saline irrigation. Stone fragments were collected and sent for C\&S.

For obtaining stones for culture we followed the technique described by Nemoy and Stamey, which is done by washing off surface contaminants and subsequently culturing bacteria with the stone. ${ }^{4}$ Stone fragments were washed in 5 sequential bottles containing sterile saline and then crushed in the fifth bottle, of which the contents were sent as stone C\&S. CPS ID3 (bioMerieux, Marcy I'Etoile, France), a chromogenic medium is used for urine C\&S, whereas crushed stones were cultured on chocolatized blood agar, aerobic blood agar, broth agar plates and anaerobic blood agar.

In all patients nephrostomy tube was clamped and removed after 24 hours. Sepsis, fever and pain following nephrostomy clamping necessitated retaining it for further period of free drainage until the resolution of these symptoms or until antegrade nephrostograms showed free drainage from the collecting system concerned. Patients were monitored closely in the postoperative period to watch for signs of systemic inflammatory response syndrome (SIRS), which is defined as the development of 2 of 4 criteria, namely temperature less than 36C or greater than $38 \mathrm{C}$, heart rate greater than 100 beats per minute, respiratory rate greater than 20 breaths per minute and white cell count greater than $12 \times 10^{9} / 1$ or less than $4 \times 10^{9} / 1$. The development of hypotension below a systolic blood pressure of $90 \mathrm{~mm}$ of $\mathrm{Hg}$ or $40 \mathrm{~mm}$ of $\mathrm{Hg}$ below baseline for the patient in the presence of features of SIRS was considered as septic shock. SIRS or shock in this study was defined as urosepsis. Blood was cultured on standard aerobic and anaerobic culture medium. The blood culture medium contained TSB broth. The data collected were devided into 3 main groups, such as MSU C\&S, pelvic urine C\&S (urine proximal to the obstructing stone) and stone C\&S. 
We performed statistical analysis of the data obtained using the Fisher exact and Pearson chi-square tests to determine associations among the various groups and subgroups. The t test was used to compare mean stone size among the subgroups analyzed. Sensitivity, specificity, positive predictive value (PPV), negative predictive value (NPV) and association risks were calculated. Univariate analysis was used to determine the association between SIRS and other parameters. A literature search was done in Index Medicus / MEDLINE to look for comparable information.

\section{Results:-}

Based on our selection criteria, total of 135 patients were recruited. Table 1 lists patient and stone demographics. Four patients required 2 percutaneous tracks to achieve complete stone clearance. No patient is this study required blood transfusion or had hypovolemia due to blood loss during procedure.

Stone C\&S was the most prevalent culture positive specimen and it was significantly higher than bladder urine C\&S (OR 45.87, fig 1). Most infected samples of pelvic urine grew Escherichia coli, whereas bladder urine grew mixed growth, most of the time when the specimen was infected (fig.2).

Table 1:- Patient and stone demographics

\begin{tabular}{|c|c|}
\hline \multicolumn{2}{|l|}{ Age } \\
\hline Range & $21-68$ \\
\hline Mean & 44.89 \\
\hline SD & 11.71 \\
\hline \multicolumn{2}{|l|}{ No. sex } \\
\hline Male & 116 \\
\hline Female & 19 \\
\hline \multicolumn{2}{|c|}{ Stone size $(\mathrm{mm})$} \\
\hline Range & $20-45$ \\
\hline Mean & 32 \\
\hline \multicolumn{2}{|c|}{ Operative time(mins) } \\
\hline Range & $30-180$ \\
\hline
\end{tabular}

None of the specimen collected from the opposite pelvicaliceal system(PUC 3) were culture positive and the results of pelvic urine $\mathrm{C} \& \mathrm{~S}$ were same for urine collected from ipsilateral side by two methods, ie, by using ureteral catheter(PUC 2) and by initial puncture(PUC 1). Hence we are considering PUC 1 only for statistical analysis.

Fig 1

\section{Culture positive specimen}

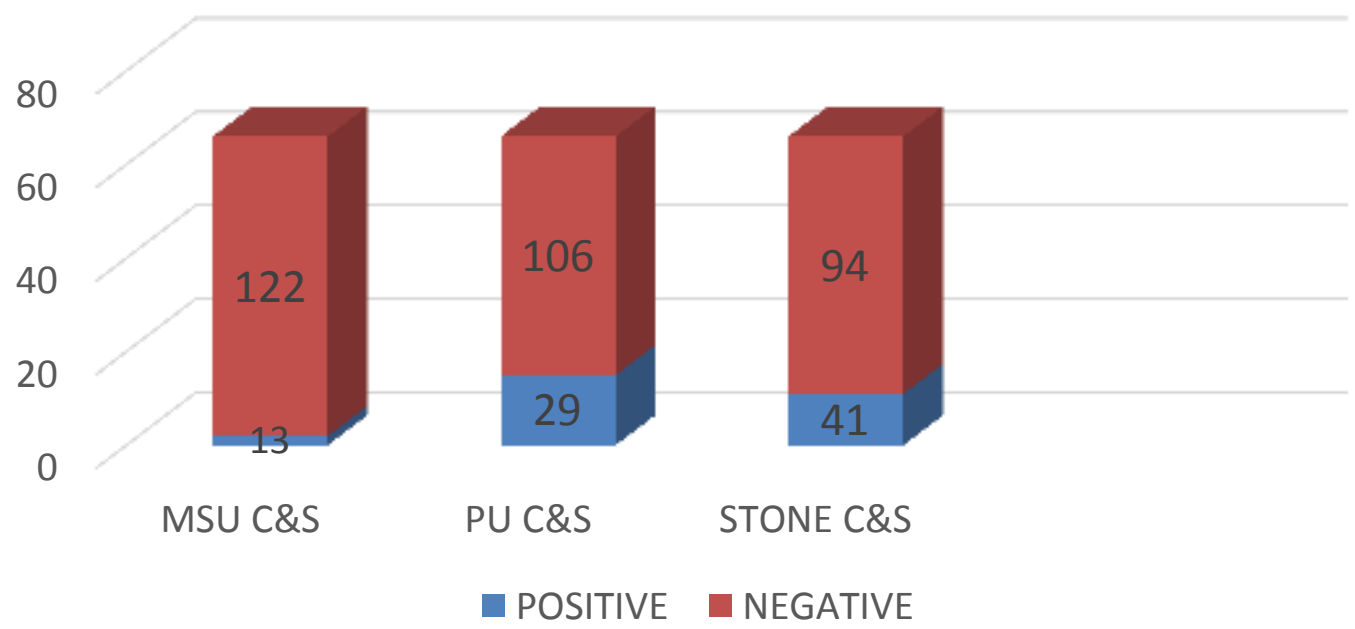


Fig 2

\section{Types of organism cultured}

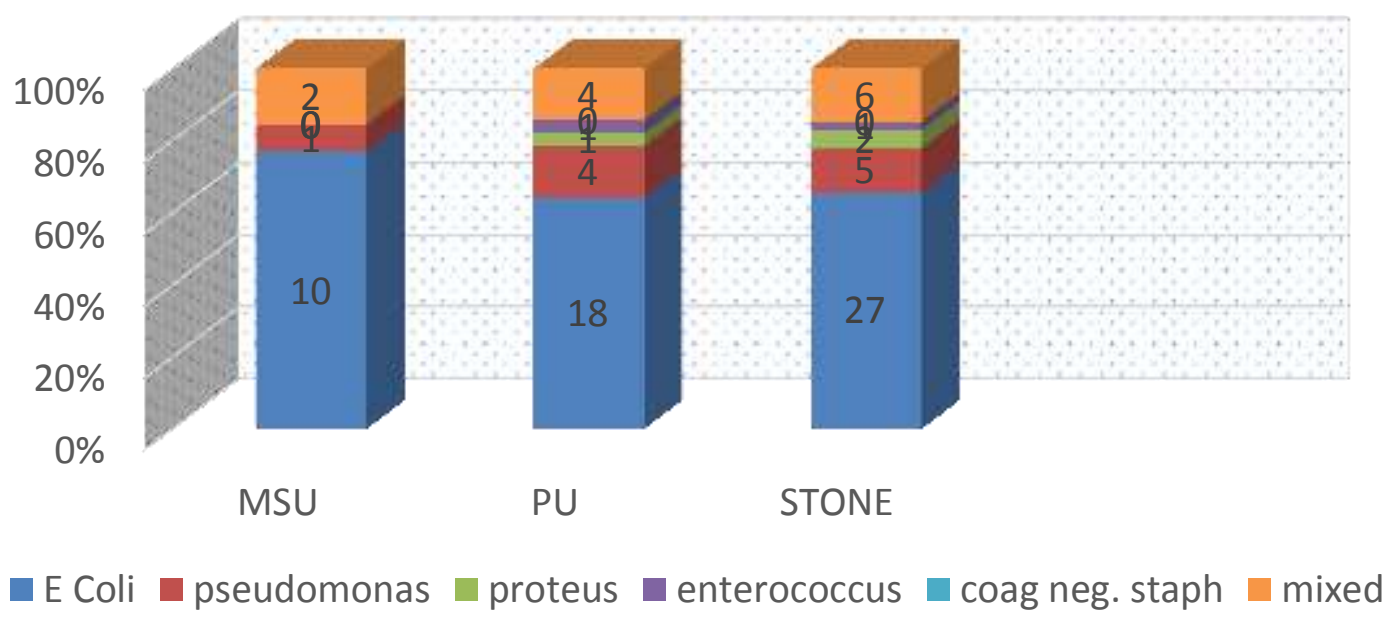

All 3 specimens were simultaneously culture positive in only 3 patients (2.22\%) (table 2). The pelvic urine and / or stone (upper tract) were infected in 70 patients (51.85\%) and bladder urine was culture positive in only $13(9.63 \%)$. This gave urine from the bladder a PPV, NPV and RR of 0.5, 0.56 and 1.15 (95\% CI 0.48 to 2.73), respectively, for detecting upper tract infection.

Table2:-

\begin{tabular}{|l|cc|}
\hline & \multicolumn{2}{|c|}{ Bladder urine culture } \\
\cline { 2 - 2 } & Positive \\
\hline Stone culture positive & & 22 \\
\hline Pelvic urine culture positive & 3 & 14 \\
\hline Pelvic urine culture negative & & 2 \\
\hline Stone culture negative & 1 & 81 \\
\hline Pelvic urine culture positive & 10 & 2 \\
\hline Pelvic urine culture negative & \\
\hline
\end{tabular}

We used bladder and pelvic urine to predict infection in stones, as infected stones were highly prevalent. Of the 2 specimens, pelvic urine was the more accurate with a 2-fold risk of being associated with infection in the stone. SIRS was seen in 43 patients (31.9\%), of whom 18 had at least 1 culture positive specimen. Features of septic shock developed in 4 patients (3\%). There were no deaths in our study group. No patients had positive blood C\&S. Correlation between the various specimens and SIRS revealed that infected stone C\&S and pelvic urine C\&S carried several fold risk of urosepsis (table 5, fig. 3).

Table5:-

\begin{tabular}{|l|c|c|c|}
\hline & Bladder urine C\&S & Pelvic urine C\&S & Stone C\&S \\
\hline \% Sensitivity & 11.6 & 53.5 & 79.1 \\
\hline \% Specificity & 91.3 & 93.5 & 92.4 \\
\hline PPV & 38.5 & 79.3 & 82.9 \\
\hline NPV & 68.9 & 81.1 & 90.4 \\
\hline RR(95\%CI) & 1.382 & 16.483 & 45.873 \\
\hline P Value & 0.591 & $<0.0001$ & $<0.0001$ \\
\hline
\end{tabular}


Fig.3

\section{Correlation between SIRS and various specimens collected}

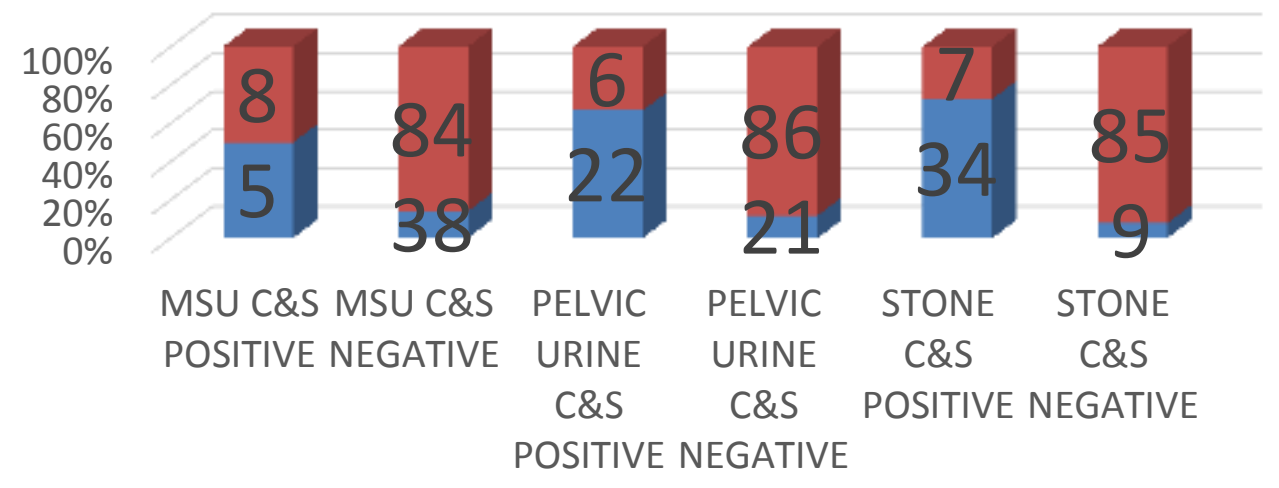

- SIRS - NO SIRS

Radiological evidence of a dilated pelvicaliceal system, that is hydronephrosis, caliectasis or a caliceal diverticulum, was seen in a total of 52 patients and the incidence of positive pelvic urine $C \& S$ in this subgroup was significantly higher than in those without obvious dilatation $(\mathrm{p}=0.046)$. Hydronephrosis did not correlate with SIRS ( $\mathrm{p}=0.529)$. Operative time had a positive linear relationship with stone bulk $(\mathrm{r}=0.723, \mathrm{p}=0.01)$, although neither of these factors correlated with urosepsis ( $\mathrm{p}=0.362$ and 0.504 , respectively). Infected stones appeared larger than non infected ones.

\section{Discussion:-}

It has been standard practice at our institute to test urine for infection at least ten days prior to PCNL by MSU C\&S. Patients with infection are treated with appropriate antibiotics for 7 days and urine C\&S repeated after this. Antibiotic prophylaxis is started in accordance with European Association of Urology (modified from infectious Diseases Society of America, and European Society of Clinical Microbiology and Infectious Diseases) guidelines. ${ }^{5}$ Sometimes systemic and catastrophic infection developed despite of this careful preoperative care. Urosepsis and shock following PCNL have been observed to occur in direct proportion to the duration of the procedure, urine bacterial load, severity of obstruction by stone and infection in the stone. ${ }^{3}$ O'Keefe et al retrospectively reviewed a series of 700 patients undergoing upper tract manipulation in their study. Severe septicemia developed in 9 patients and $66 \%$ died. In the same centre Rao et al observed minor forms of septicemia in $37 \%$ of 27 patients undergoing PCNL. A stark comparison to this is the series of Charton et al of 216 patients who underwent PCNL with no prophylactic antibiotics and no major septic complications, in spite of $35 \%$ having infected urine preoperatively. ${ }^{6}$

In our study retrograde contamination of renal pelvic urine was excluded since bladder urine specimens were negative in all patients with infected pelvic urine and there was a clear discrepancy between the types of microorganisms cultured. The sterile techniques used in the study and rigorous washing of stones ensured that no cross - contamination occurred and thus did not affect the isolation of organism.

Those culturing stones using the Nemoy and Stamey method have described the incidence of infected stones ${ }^{4}$ as $5.6 \%$ to $77.3 \% .^{7-11}$ In Canada Grault et al attributed the $5.6 \%$ rate of infected stones to a longer period of preoperative antibiotics with fluoroquinolones and the lower prevalence of noninfective stones. ${ }^{7}$ Fowleer, who reported a stone positive rate of $77.3 \%$, found that urine C\&S was simultaneously positive in only $12.5 \%$ of patients with infected stones $^{12}$ (our rate was $10.5 \%$ ).

With regard to infected pelvic urine Pode et al catheterized 135 upper tracts with stones and isolated infected pelvic urine in $75.6 \%$. The higher incidence could be explained by the fact that they included patients with obstructing upper tract stones. Stones were not cultured in this series. In 1984 Lewi et al analyzed stones, pelvic urine and 
bladder urine from 63 patients, and found that urine C\&S was positive in $29 \%$, stones were infected in $38 \%$ and pelvic urine was infected in $30 \% .{ }^{13}$ Mariappan and Loong also corroborated this finding in their series of ureteroscopies in Malaysia, where in $58.6 \%$ of cases pelvic urine was infected. ${ }^{14}$ They also confirmed the correlation between stone C\&S and pelvic urine C\&S with the 2 specimens having identical microorganisms twothirds of the time.

Preoperative and postoperative changes in endotoxin and tumor necrosis factor (TNF) were described in their series by Rao et al. ${ }^{2}$ Only 27 of the 117 patients in their study underwent PCNL. Of these patients $74 \%$ had fever, $41 \%$ had increased TNF, $41 \%$ had endotoxemia and $37 \%$ had bacteremia. Endotoxins were found to be present in serum even before operative manipulation. Although stones were infected in $47.8 \%$ of cases, which was most closely related to bacteremia, the incidence of preoperative bacteriuria in patients undergoing PCNL was not mentioned. Bacteriuria had a PPV of 0.53 for detecting endotoxemia when patients with nephrostomies, ureteropyeloscopy, stenting and prior shock wave lithotripsy were all included. A sensitivity and specificity of 54\% and $78 \%$, respectively, appeared to follow a trend similar to our findings. The correlation between stone C\&S and pelvic urine C\&S with TNF and endotoxin levels has alredy been observed. Blood C\&S in our series did not prove to be an accurate test even in the face of septic shock. Martin et al observed that only $50 \%$ of patients with overt sepsis had bactermia. $^{15}$

We used a clinical definition of SIRS to describe sepsis, as recommended by the Sepsis definition consensus committee in 1992, which was reviewed and accepted at the 2001 consensus meeting. ${ }^{16}$ Macdonald $^{17}$ and Cadeddu et $\mathrm{al}^{18}$ also confirmed no correlation between operative time and postoperative fever. Cadeddu et al from the Johns Hopkins restrospectively reviewed 66 records of patients in whom fever with temperature greater than 38C developed: none had positive blood $\mathrm{C} \& \mathrm{~S}$ or postoperative urine $\mathrm{C} \& \mathrm{~S}$. Caddedu et al found no correlation between fever and stone composition. Stone culture was not performed in their study. Fever alone cannot be used as an indicator of systemic infection, as noted in the study of Rao et al, in which $74 \%$ of Patients with PCNL had fever postoperatively, of which only $41 \%$ had endotoxemia. ${ }^{2}$

Although the definitions vary, septic shock rates have been reported to be $1 \%$ to $2 \% .{ }^{19}$ While the incidence of septic shock in our series is higher, this probably reflects our tertiary referral practice, which deals with complex stones. It would seem advisable to specify the proportion of patients with an abnormal drainage system when judging sepsis rates.

Larger stones are more likely to be triple phosphate stones and they have been found to harbour infection. Szigeti et al found infected stones in $10 \%$ of their 57 patients with renal stones and they noted that bacteriuria was more prevalent in stones greater than $30 \mathrm{~mm}$ in diameter. ${ }^{20}$ Although in our series stone bulk did not directly correlate with uro sepsis, more of the larger stones(greater than $20 \mathrm{~mm}$ ) were infected. We also noted that the turbidity of urine at pelvicaliceal system puncture did not correlate with SIRS and positive Pelvic urine because only $50 \%$ of the patients with cloudy urine had infected stones or positive pelvic urine culture. To our knowledge there are no published article providing similar information with regard to a correlation between urine turbidity and urosepsis. Similarly there is no literature available that attempts to correlate hydronephrosis, age, sex, residual stones and urosepsis in the Index Medicos/ MEDLINE.

Because many patients with renal stones, especially struvite, have been treated for recurrent urinary tract infections, the potential for antibiotic resistance becomes high. Empirical antibiotic therapy will salvage the situation in most instances. However, we believe that samples collected from the upper tract will be the best guide to therapeutic antibiotic use when systemic infection arises, as indicated in this series by the patient with Actinomyces in the pelvic urine sample, in whom sepsis developed and who would not have received appropriate antibiotics in the absence of this sample.

The preoperative prediction of urosepsis is ideal and some groups at high risk can be identified, such as patients, with abnormal anatomy, stag horn calculi, diabetes, and immune compromised patients. We also identified large stones and dilated pelvicaliceal systems as possible predictors of urosepsis in this series. This has enabled us to produce a department protocol to administer antibiotics to these patients for 1 week prior to surgery. It may also be judicious to aspirate pelvic urine percutaneously in patients who are more prone to develop sepsis, like those with and ileal conduit or dilated pelvicaliceal system and immunocompromised, and treat according to culture report. A second puncture for better stone clearance per se did not increase the incidence of sepsis or blood loss in this study. 


\section{Conclusions:-}

The results of this study suggest that stone $\mathrm{C} \& S$ and pelvic urine $C \& S$ are better predictors of urosepsis than bladder urine $\mathrm{C} \& \mathrm{~S}$ in patients undergoing PCNL. There is no need to collect urine from opposite pelvicaliceal system for C\&S. A dilated pelvicaliceal system and stones larger than $20 \mathrm{~mm}$ predicted patients with infected pelvic urine and infected stones, respectively. We recommed that consideration should be given to the pretreatment of patients with dilated pelvicaliceal systems and stones $20 \mathrm{~mm}$ or greater with a broad spectrum antibiotic for one week, and the collection of stones and pelvic urine for culture and sensitivity.

\section{Appendix: Exclusion Criteria:-}

1. Patients with a stent, nephrostomy tube or indwelling catheter

2. Diabetes mellitus

3. Renal failure

4. Fever prior to surgery

5. Previous manipulation/procedure

6. Concomitant bladder stone or tumor

7. Patients on therapeutic antibiotics

8. Contra lateral renal/urethral stone

\section{References:-}

1. O'Keeffe, N.K.Mortimer, A.J.Sambrook, P.A. and Rao, P.N: severe sepsis following percutaneous or endoscopic procedures for urinary tract stones, Br J Urol, 72: 277,1993

2. Rao, P.N. Dube, D.A. Weightman, N.C., Oppenheim, B.A. and Morris, J ;Prediction of Septicemia following endourological manipulation for stones in the upper urinary tract. J Urol,146: 955,1991

3. Stamey, T.A: Pathogenesis and Treatment Urinary Tract Infections, Baltimore: Williams \& Wilkins Co.., chapt.8,p,430 1980.

4. Nemoy, N.J. and Stamey, T.A: surgical, bacteriological and biochemical management of "INFECTION STONES.” JAMA,215: 1470,1971.

5. European Association of Urology (EAU) Guidelines on Urinary Tract Infection, 2001 ISBN 90-806179-3-8, and published online. Available at www.uroweb.org. Accessed December 2003

6. Charton.M. Vallancien, G.Veillon,B. and Brisset, J,M: Urinary tract infection for percutaneous surgery for renal calculiJ Urol,135: 15,1986.

7. Gault, M.H. Longerich, L.L.Crane, G.,Cooper, R., Dow,D.,Best, L et al: Bacteriology of urinary tract stones, J Urol,153: 1164,1995.

8. Fowler, J.E Jr.Bacteriology of branched renal calculi and accompanying urinary tract infection. J Urol, $131: 213,1984$

9. MeCartney, A.C. Clark, J.and Lewo. H.J.: Bacteriological study of renal calculi. Eur J Clin Microbiol, 4: 553, 1985.

10. Bratell, S., Broson, J.E., Grenabo,L., Hedelin, H. and Perttersspm, S.,The bacteriology of operatied renal stones, Eur Urol.17:58,1990.

11. Hugossson, J., Grenabo, L., Hede;om. H., Petterson, S. and seeberg. S:Bacteriology of upper urinary tract stones, J Urol,143: 965, 1980.

12. Pode, D., Lenkovsky, Z., Shapiro, A. and Pfau, A: can extractor poreal shock wave lithtripsy eradicate persistent urinary infection associated with infected stones? J Urol,140: 257,1988

13. Lewi, H.J.,White, A., Hutchinson, A.G. and Scott, R: the bacteriology of the urine and renal calculi. Urol Res, 12:107, 1984.

14. Mariappan, $\mathrm{P}$ and Loong, $\mathrm{C}, \mathrm{W}$ : Midstream urine culture and sensitivity test is a poor predictor of infected urine proximal to the obstructing ureteric stone or infected stone : as a prospective clinical study.J Urol, 171: $2142,2004$.

15. Martin,G.S., Mannino, D.M., Eaton, S. and Moss, M: The epidemiology of sepsis in the United States from 1979 through 2000, N Engal J Med, 348: 1546, 2003.

16. Levy, M.M., Fink,M.P, Marshall, J.C.Abraham.E., Angus., D., Cook., D., Cook, D.et al: 2001 SCCM/ESICM/ACCP/ATS/SIS International Sepsis Definitions Conference. Crot Care Med. 31: 1250. 2003.

17. Macdonald, J: Percutaneous nephrostolithtomy for staghorn calculi: maximum operation duration for optimal outcome. Aust NZ J surg.70: A165, 2000. 
18. Cadeddu, J.A., Chen, R.,Bishoff, J.,Micali,S.,Kumar, A.,Moore, R.G et al: clinical significance of fever after percutaneous nephrostolithotomy. Urology.52:48,1998.

19. Segura, J.W., Preminger, G.M., Assokos, D.G.Dretler, S.P.khan. R.I Lingeman, J.E et al : Nephrolithotomy . Urology,52: 48,1998.

20. Shigeta, M., Hayashi, M. and lgawa,M:A clinical study of upper urinary tract calculi treated with extracorporeal shock wave lithoripsy : association with bacteriuria before treatment. Urol Int, 54:214, 1995. 